\title{
Imaging of 2-mm dust continuum emission towards S106 FIR and its spectral energy distribution
}

\author{
Ray S. Furuya \\ Osservatorio Astrofisico di Arcetri, Largo Enrico Fermi 5, 50125, \\ Firenze, Italy \\ Yoshimi Kitamura \\ Institute of Space and Astronautical Science, Yoshinodai, Sagamihara, \\ Kanagawa 229-8510, Japan \\ Alwyn Wootten \\ National Radio Astronomy Observatory, 520 Edgemont Road, \\ Charlottesville, VA 22903, USA \\ Mark J. Claussen \\ National Radio Astronomy Observatory, 1003 Lopezville Road, Socorro, \\ NM 87801, USA \\ Ryohei Kawabe \\ Nobeyama Radio Observatory, Nobeyama 411, Minamimaki, \\ Minamisaku, Nagano 384-1305, Japan
}

\begin{abstract}
The class 0 source S106 FIR is a good candidate to investigate a very early phase of star formation because of the presence of an AU-scale Microjet, discovered by our VLBA $\mathrm{H}_{2} \mathrm{O}$ maser observations and the absence of an extensive $\mathrm{CO}$ outflow. In order to reveal the properties of S106 FIR, we conducted observations of 2-mm continuum emission with the Nobeyama Millmeter Array. We detected a weak compact continuum emission around S106 FIR. We analysed the spectral energy distribution of S106 FIR. It is found that the dust temperature and the $\beta$ index of the dust opacity range from 31 to $55 \mathrm{~K}$ and from 1.4 to 1.6 , respectively. Using these results, we computed the mass of the core aound S106 FIR and the bolometric luminosity of 3.1 to $5.8 M_{\odot}$ and 230 to $1070 L_{\odot}$, respectively.
\end{abstract}

\section{Backgrounds}

We discovered a newly ejected extraordinarily compact protostellar jet - a Microjet - from the class 0 protostar S106 FIR $(d=600 \mathrm{pc})$ through VLA observations of $\mathrm{H}_{2} \mathrm{O}$ masers (Furuya et al. 1999). Subsequent sub-AU resolution VLBA observations revealed the presence of a U-shaped distribution of the 
masers, located at $25 \mathrm{AU}$ from the star, in the center of the cloud core (Furuya et al. 2000). The U-shaped distribution is considered to be a "micro bowshock" created by the impact of the Microjet on the ambient cloud medium. In addition, this source stands unique among all 38 known low-mass class 0 sources in its lack of an extensive $\mathrm{CO}$ outflow, evidenced by our sensitive multi-transition CO searches with $10 \sigma$ upper limits of $\sim 10^{-6} M_{\odot}$ per $\mathrm{km} \mathrm{s}^{-1}$.

We suggest that S106 FIR is the first protostar discovered in an evolutionary stage before the formation of a $\mathrm{CO}$ outflow because of the presence of the Microjet and the absence of a large scale CO outflow. The youth of S106 FIR is supported by the following age estimate. One estimate is provided by the nondetection of the ${ }^{12} \mathrm{CO} J=3-2$ wing emission within the CSO beam. This gives an upper limit to the dynamical time scale of the outflow. It takes $\sim 900$ years for the Microjet to move across the radius of the CSO beam with a velocity of $35 \mathrm{~km} \mathrm{~s}^{-1}$ derived from our VLBA proper motion study. Another estimate of the age is as follows: the ratio of class 0 sources without outflows to those with outflows $\left(\frac{1}{40}\right)$ multiplied by the lifetime of the class 0 stage of $\lesssim 10^{4}$ years argues an age of $\lesssim 250$ years. These results have been already published (Furuya et al.2000), and here we report our new results of interferometric continuum imaging of S106 FIR.

\section{Observations}

Continuum emission imaging at 2-mm was performed with the Nobeyama Milimeter Array (NMA) of the Nobeyama Radio Observatory ${ }^{1}$ in 1998-1999 using three different array configurations. The phase tracking center was set at the position of the $\mathrm{H}_{2} \mathrm{O}$ masers, and the field of view was $51^{\prime \prime}$. The projected baselines ranged from 2.9 to $45 \mathrm{k} \lambda$ : our observations were insensitive to structures extended more than $31^{\prime \prime}(\sim 19,000 \mathrm{AU})$. The resulting synthesized beam size was $1.6^{\prime \prime} \times 1.5^{\prime \prime}$ at P.A. $=-31^{\circ}$. The Ultra Wide Band Correlator was employed with $1 \mathrm{GHz}$ bandwidth centered at $146.96 \mathrm{GHz}$. The phase and gain calibrator was $2145+067$, and the bandpass calibrator 3C273. The flux density of $2145+067$ was derived from observations of the Uranus, and we estimated uncertainty of flux calibrations to be less than $30 \%$. We used the software package AIPS to make a CLEAN image with natural weight. The rms noise level of the image was $7.8 \mathrm{mJy} \mathrm{beam}^{-1}$.

\section{Results}

Figure 1 indicates a map of the continuum emission around S106 FIR at 2 $\mathrm{mm}$. Although Richer et al.(1993) reported that S106 FIR was identified as a bright point source with a diameter of $6^{\prime \prime}(3600 \mathrm{AU})$ at $450 \mu \mathrm{m}$, such a centrally condensed compact dust emission cloud not be detected by our interferometric imaging. We marginally detected a weak emission extended over a 2500

\footnotetext{
${ }^{1}$ Nobeyama Radio Observatory (NRO) is a branch of National Astronomical Observatory, an interuniversity research institute operated by the Ministry of Education, Science, and Culture of Japan
} 

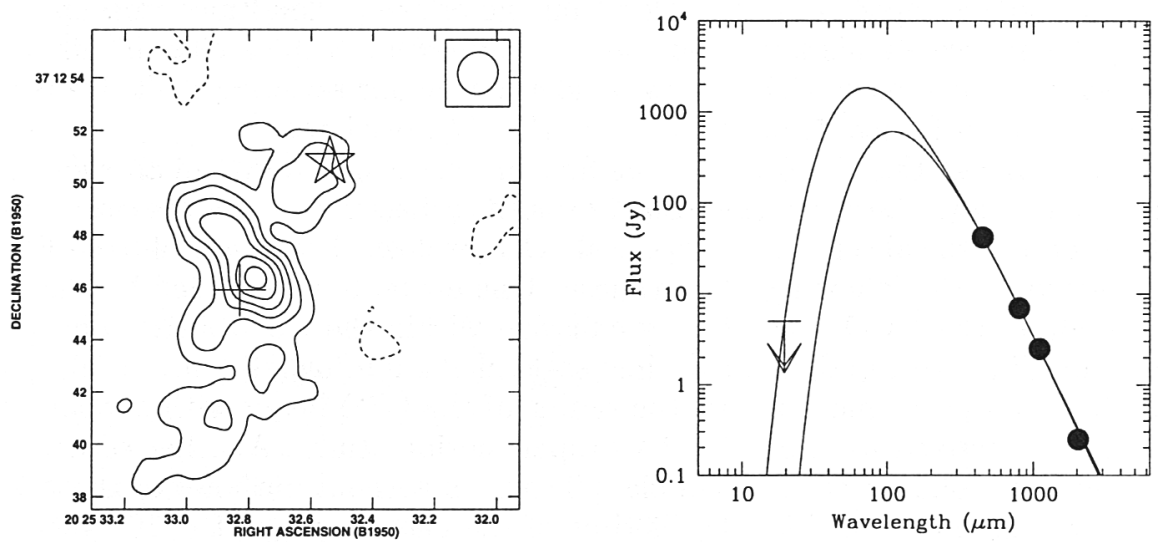

Figure 1. (left) Image of the 2-mm continuum emission around S106 FIR observed by the NMA. The star and cross indicate the position of the masers and the peak position of the $5 \mathrm{GHz}$ continuum emission (Felli et al. 1984), respectively. The contour intervals are $2 \sigma$, starting at $3 \sigma$ level $\left(1 \sigma=7.8 \mathrm{mJy}\right.$ beam $\left.^{-1}\right)$. To the right, the spectral energy distribution of S106 FIR.

AU $\times 2300 \mathrm{AU}$ area at the southwest of the masers. The total flux density of the emission is $152 \pm 16 \mathrm{mJy}$. Since in our $1.5^{\prime \prime}$ resolution NMA image, the source was partially resolved, we convolved this map with $3^{\prime \prime}$ beam using all the array configuration data. Then we obtained the flux density of $248 \pm 22 \mathrm{mJy}$. We could not convolve with a larger beam than $3^{\prime \prime}$ because of the contamination of the bight rim of the nearby HII region.

\section{Analysis of the SED and Discussion}

S106 FIR is neither visible in the optical nor in the near-infrared. However it was identified as a point source at $450 \mu \mathrm{m}$, and flux densities were measured at 450,850 , and $1100 \mu \mathrm{m}$ with the JCMT (Richer et al. 1993). Gehrz et al.(1982) provides an upper limit of $19.5 \mu \mathrm{m}$ for the flux density at $2 \mathrm{~mm}$.

We analysed the SED of S106 FIR in terms of a single-temperature greybody: all the emission at 2-mm is assumed to come from dust with a single temperature. In this case we can fit the spectrum to a greybody form of $S_{\nu}=B_{\nu}\left(T_{\text {dust }}\right)\left[1-\exp \left(-\tau_{\nu}\right)\right] \Delta \Omega$, where $B_{\nu}\left(T_{\text {dust }}\right)$ is the Planck function at a frequency $\nu$ with a dust temperature $T_{\text {dust }}, \tau_{\nu}\left(=\kappa_{\nu}\langle\Sigma\rangle\right)$ is the optical depth due to the dust opacity, and $\Delta \Omega$ is the source solid angle. Here $\kappa_{\nu}$ is the dust opacity coefficient, and $\langle\Sigma\rangle$ is a mean mass column density. We assume that $\kappa_{\nu}$ values as $\kappa_{0}\left(\nu / 10^{12} \mathrm{~Hz}\right)^{\beta}$ where $\beta$ is an index of the dust emissivity, and for $\kappa_{0}$, we adopted the widely accepted Hildebrand value (Hildebrand 1982). 


\begin{tabular}{lllllr}
\hline \multicolumn{7}{c}{$R=1800 \mathrm{AU}$} & (Richer et al. 1993) & & \\
& $T_{\text {dust }}$ & $\langle\Sigma\rangle$ & $\beta$ & $M$ & $L_{\text {bol }}$ \\
& $(\mathrm{K})$ & $\left(\mathrm{g} \mathrm{cm}^{-2}\right)$ & & $\left(M_{\odot}\right)$ & $\left(L_{\odot}\right)$ \\
upper limit at $19.5 \mu \mathrm{m}$ & 55 & 2.7 & 1.4 & 3.1 & $\leq 889$ \\
no upper limit at $19.5 \mu \mathrm{m}$ & 40 & 3.8 & 1.5 & 4.3 & 273 \\
\hline$R=5300 \mathrm{AU}$ & (Furuya et al. 2000, see Fig. 1) & \\
\multicolumn{7}{c}{} \\
upper limit at $19.5 \mu \mathrm{m}$ & 48 & 0.36 & 1.4 & 3.5 & $\leq 1070$ \\
no upper limit at $19.5 \mu \mathrm{m}$ & 31 & 0.59 & 1.6 & 5.8 & 230 \\
\hline
\end{tabular}

Table 1. Summary of SED Analysis of S106 FIR.

In order to obtain $T_{\text {dust }},\langle\Sigma\rangle$, and $\beta$, we fitted the data using the greybody equation $S_{\nu}=\pi(R / d)^{2}\left(2 h \nu^{3} / c^{2}\right)\left[1-\exp \left(-\langle\Sigma\rangle \kappa_{0} \nu^{\beta}\right)\right] \times\left[\exp \left(h \nu / k T_{\text {dust }}\right)-1\right]^{-1}$ For the core radius $R$, we used the following two values: 1800 AU from the 450 $\mu \mathrm{m}$ map (Richer et al. 1993) and $5300 \mathrm{AU}$ from our $\mathrm{H}^{13} \mathrm{CO}^{+} J=1-0$ map with the NMA (Furuya et al. 2000). We found from the above model fitting that the dust temperature, the mean mass column density, and the $\beta$-index range from 31 to $55 \mathrm{~K}$, from 0.36 to $3.8 \mathrm{~g} \mathrm{~cm}^{-2}$, and 1.4 to 1.6 , respectively (Table 1 ). The derived $\beta$-index of around 1.5 agrees with the characteristic values of $1.5 \sim 2.0$ for class 0 sources (e.g., Andrè et al. 1993; Ward Thompson et al. 1995b). Moreover, these results are consistent with the previous analysis by Richer et al.(1993). Using these model fitting results, we calculated the mass of the core around S106 FIR given by $\pi\langle\Sigma\rangle R^{2}$. When we adopted $R=5300 \mathrm{AU}$, the derived mass ranges from 3.5 to $5.8 M_{\odot}$, consistent with our measurement of $4.2 M_{\odot}$ from $\mathrm{H}^{13} \mathrm{CO}^{+} J=1-0$ observations (Furuya et al. 2000). We also computed the bolometric luminosity $\left(L_{\text {bol }}\right)$ of 230 to $1070 L_{\odot}$ by integrating Eq.(1). Despite the low outflow activity (Furuya et al. 2000), the protostar is very luminous: this could result from a rapid accumlation of protostellar mass in the early accretion phase (Masunaga and Inutsuka 2000).

\section{References}

Andrè, P., Ward-Thompson, D., \& Barsony, M. 1993, ApJ, 406, 122

Felli, M. et al. 1984, A\&A, 135, 261

Furuya, R. S. et al. 1999 ApJ, 525, 821

Furuya, R. S. et al. 2000 ApJ, 542, L135

Gehrz, R. D. et al. 1982 ApJ, 254, 550

Hildebrand, R. H. 1983 QJRAS, 24, 267

Masunaga, H. \& Inutsuka, S. 2000 ApJ, 531, 350

Richer, J. S. et al. 1993 MNRAS, 262, 839

Ward-Thompson, D. et al. 1995 MNRAS, 274, 1219 